\title{
A Simple Approach to Compute Interatomic Force Constant for Mono and Diatomic Semiconductors
}

\author{
Mohammed Joghlaf' ${ }^{1}$, Yahya Ababou' ${ }^{1}$, Salaheddine Sayouri ${ }^{2}$ \\ ${ }^{1}$ LPAIS Department of Physics, Faculty of Science, Fez, Morocco \\ ${ }^{2}$ BP Sidi Brahim, Fez, Morocco \\ Email:mhjoghlaf@gmail.com, ababouyahya@hotmail.com, ssayouri@gmail.com
}

How to cite this paper: Joghlaf, M., Ababou, Y. and Sayouri, S. (2021) A Simple Approach to Compute Interatomic Force Constant for Mono and Diatomic Semiconductors. Journal of Applied Mathematics and Physics, 9, 11-20.

https://doi.org/10.4236/jamp.2021.91002

Received: November 25, 2020

Accepted: January 2, 2021

Published: January 5, 2021

Copyright $\odot 2021$ by author(s) and Scientific Research Publishing Inc. This work is licensed under the Creative Commons Attribution International License (CC BY 4.0).

http://creativecommons.org/licenses/by/4.0/

\begin{abstract}
In this paper, a mathematical relation was found between interatomic Hooke's force constant and both the bulk modulus and interatomic distance in solid crystals, considering that the forces which have effect on an atom are only those resulted from the neighboring atoms, and the forces are subject to Hooke's law as the deflections of atoms from their equilibrium positions are very small. This work has been applied on some solid semiconducting crystals of diatomic primitive cell, including crystals of mono-atomic primitive cell automatically, by using linear statistical fitting with computer programming and, then, using mathematical analysis, proceeding from the vibrational dispersion relation of solid linear lattice, these two methods have been used in the process in order to support each other and for the result to be satisfying and reasonable. This is a contribution to the process of using computer programming in physics to facilitate mathematical analyses and obtain the required relations and functions by designing and developing appropriate computer programs in line with the macro and micro natures of materials. The importance of this is in enhancing our understanding of the interatomic actions in cells and of the crystal structure of materials in general and semiconductors in particular, as it is a step of the initial steps to facilitate the process of calculating energies and extracting mathematical relations between correlation energy and temperature as well as between sub-fusion and fusion energies with temperature.
\end{abstract}

\section{Keywords}

Interatomic Force Constant, Bulk Modulus, Vibrational Dispersion Relation, Linear Fitting, Wave Speed 


\section{Introduction}

Obtaining a mathematical relation of interatomic Hooke's force constant to bulk modulus and interatomic distance in solids, especially in semiconductors, is very important as a relation connecting macroscopic and microscopic quantities that helps in understanding the interaction between atoms and calculating energies and other quantities. Dedicated bond force constant and bulk modulus of $\mathrm{C} n$ fullerenes $(n=20,28,36,50,60)$ are computed using density functional theory (DFT) and finite element analysis (FEA) by Peon et al. The bond force constants predicted by DFT are then used as an input for finite element analysis (FEA) of the fullerene [1]. Santiago-Perez et al. obtained the linear chain equations that should be used to obtain the phonon dispersion relations along high symmetry directions from the 3D bulk problem [2]. Other work by Kot et al. found that there is a well-defined relationship between the geometric characteristics of the mass spring model systems and physical properties of the modeled materials [3], and so Dolocan et al. presented theoretical expressions relating the cohesive energy to bulk modulus, the force constant and the lattice constant applicable to solids with a variety of crystal structures, by assuming, under nearest-neighbor approximation, the interatomic force to be a polynomial of $2^{\text {nd }}$ degree $F=-\beta x+\gamma x^{2}$ [4].

In this paper, it has been assumed that Hooke's force is the only affecting interatomic force under nearest-neighbor approximation $(F=-\beta U)$, which was used to find a mathematical expression for solid crystals of diatomic primitive cell, including those of mono-atomic one automatically, relating interatomic Hooke's force constant $(\beta)$ to bulk modulus and interatomic distance by using two methods, linear statistical fitting with computer programming and mathematical analysis. We used the vibrational dispersion relation of a linear lattice of diatomic primitive cell and Data of some semiconductors (C (diamond), $\mathrm{Si}, \mathrm{Ge}$, $\mathrm{SiC}, \mathrm{ZnS}, \mathrm{ZnTe}$, CdS and CdTe).

In spite of existing other forces than Hooke's one and which may be of other atoms than the neighbors, but that do not affect much the process of calculating energies and other quantities as was shown in Einstein and Debye theories for specific heat in solids [5] [6], we tried to simplify the mathematical processing and, at the same time, obtain an accurate mathematical relation as far as possible.

In order to get the mathematical relation that we seek, we considered that the interatomic forces in a linear lattice are subject to Hooke's law with small atomic deflections as $(F=-\beta U)$, where $F$ is the Hooke's force, $U$ is small deflection and $(\beta)$ is the force constant, with neglecting other effects to facilitate the process mathematically for scientific purposes [7].

\section{Theoretical Work}

As we know, the two functions of the dispersion relation which describe linear vibrations of a diatomic primitive cell as shown in Figure 1 (the mono-atomic 
primitive cell is automatically included) are [8].

The acoustic branch

$$
\omega_{a c}^{2}=\beta\left[\left(\frac{1}{M}+\frac{1}{m}\right)-\sqrt{\left(\frac{1}{M}+\frac{1}{m}\right)^{2}-\frac{4}{m M} \sin ^{2}\left(\frac{q a}{2}\right)}\right],
$$

The optical branch

$$
\omega_{o p}^{2}=\beta\left[\left(\frac{1}{M}+\frac{1}{m}\right)+\sqrt{\left(\frac{1}{M}+\frac{1}{m}\right)^{2}-\frac{4}{m M} \sin ^{2}\left(\frac{q a}{2}\right)}\right],
$$

where $m$ and $M$ are the masses of both atoms in the primitive cell $(M \geq m)$ and $a$ is the interatomic distance. The possible deflections of two atoms are

$$
\begin{aligned}
& U(l)=U_{m} \mathrm{e}^{i(q \cdot l a-\omega t)} \quad \text { (for the bigger mass), } \\
& u(l)=u_{m} \mathrm{e}^{i(q \cdot l a-\omega t)} \text { (for the smaller mass), }
\end{aligned}
$$

where $U_{m}$ and $u_{m}$ are the maximum deflections for both atoms, $\omega$ is the angular frequency and $t$ is the time [5].

Figure 2 shows the two acoustic and optical branches functions which are separated by the prohibited $\omega$-zone.

Where the allowed values of $q$ (wave number) are given as follows:

$$
q=l \frac{2 \pi}{N a}, N \text { is the number of primitive cells in the crystal, } l= \pm 1, \pm 2, \pm 3, \cdots
$$

This means that all the solution points are within the first two Brillouin zones, so, if the number of atoms in each primitive cell is three, then all the solutions are within the first three Brillouin zones [7].

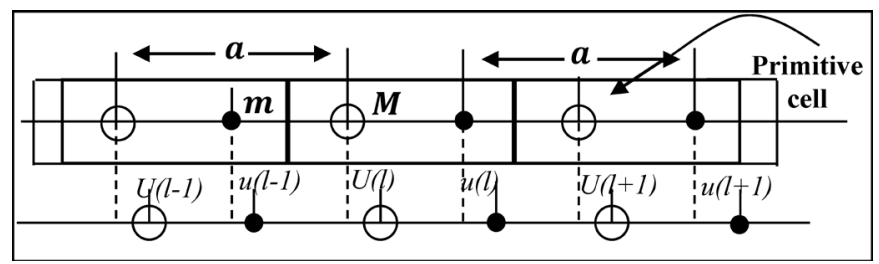

Figure 1. A linear solid lattice of diatomic primitive cell.

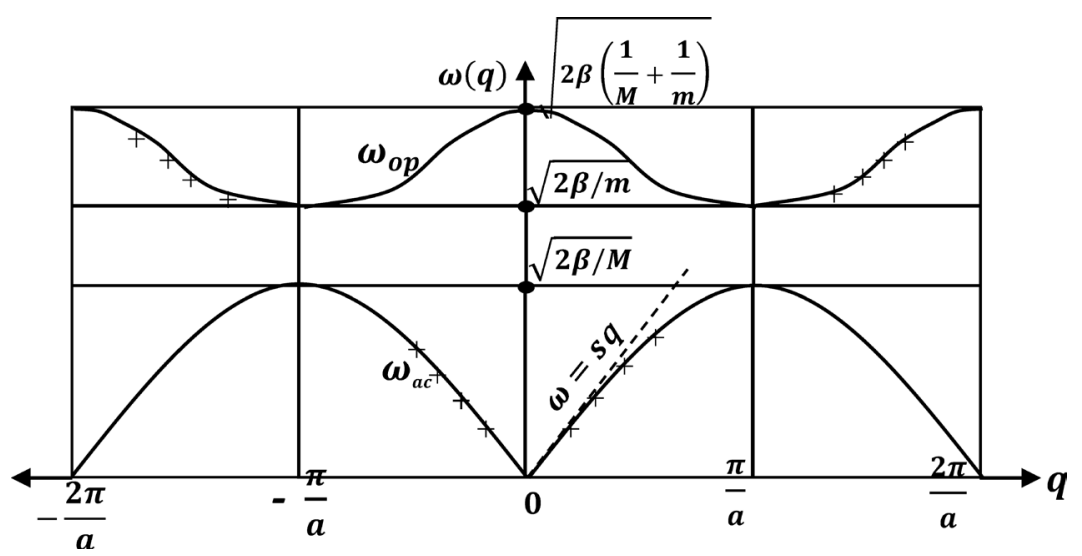

Figure 2. The two curves of dispersion relation [9]. 


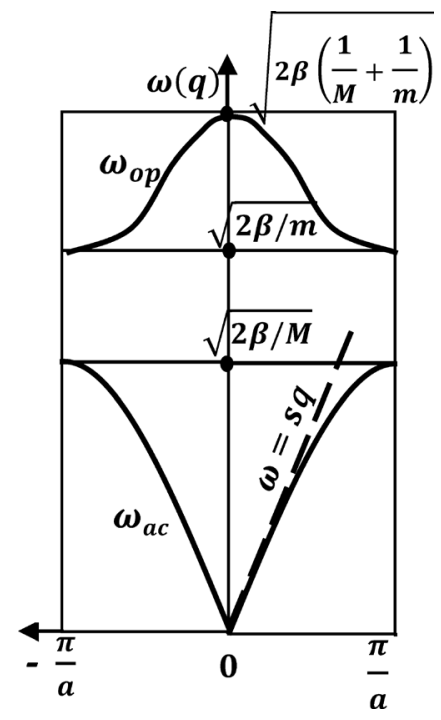

Figure 3. Approximation of dispersion relation by a straight line $\omega=s q$ beside $q=0$ [9].

We proceeded from the acoustic branch of the dispersion relation in a linear solid lattice of diatomic cell (Equation (1)) and the fact that where $q$ approaches zero, it approximately takes the following form [6]:

$$
\omega=s q \text {, }
$$

where $s$ is the wave (sound) speed via the lattice, as shown in Figure 3.

\subsection{Linearstatistical Fitting}

We should make some mathematical simplifications in Equation (1) (the acoustic branch) to remove the function dependence on the physical characteristics as follows.

Equation (1) may be rewritten under the following form:

$$
\omega^{2}=\frac{\beta}{m}\left[\left(\frac{m}{M}+1\right)-\sqrt{\frac{m^{2}}{M^{2}}+1+\frac{2 m}{M} \cos a q}\right],
$$

or

$$
\sqrt{\frac{m}{\beta}} \omega=\sqrt{\left(\frac{m}{M}+1\right)-\sqrt{\frac{m^{2}}{M^{2}}+1+\frac{2 m}{M} \cos a q}},
$$

assuming that $x=a q, \quad p=\frac{M}{m} \quad(M \geq m$, then $p \geq 1)$ and $\varpi=\sqrt{\frac{m}{\beta}} \omega$, then

$$
\varpi=\sqrt{\left(1+\frac{1}{p}\right)-\sqrt{1+\frac{1}{p^{2}}+\frac{2}{p} \cos x} .}
$$

By using a VISUAL BASIC program that generates $50(\varpi, x)$ points beside $x=0$ via substituting with values of $x$ in Equation (8) and uses them for linear statistical fitting as $\varpi=\eta_{0}+\eta_{1} x$ after calculating each of $\eta_{0}$ and $\eta_{1}$, we can reach the sought relation.

When running the program which needs to enter the values of $p=M / m$ for each matter, the $\eta_{1}$ values are as Table 1 shows. 
Table 1. Estimated values of $\eta_{1}$ for the used matters.

\begin{tabular}{ccccc}
\hline Crystal & $m\left(\times 10^{26}\right)[10]$ & $M\left(\times 10^{26}\right)[10]$ & $p=\frac{M}{m}$ & $\eta_{1}$ \\
\hline C-C & 1.994022912 & 1.994022912 & 1 & 0.495478885554644 \\
$\mathrm{Si}-\mathrm{Si}$ & 4.663788810 & 4.663788810 & 1 & 0.495478885554644 \\
$\mathrm{Ge}-\mathrm{Ge}$ & 12.05213349 & 12.05213349 & 1 & 0.4954788855554644 \\
$\mathrm{Si}-\mathrm{C}$ & 1.994022912 & 4.663788810 & 2.338884263 & 0.381690077840471 \\
$\mathrm{Zn}-\mathrm{S}$ & 5.322928773 & 10.85505562 & 2.039301310 & 0.400556205681922 \\
$\mathrm{Zn}-\mathrm{Te}$ & 10.85505562 & 21.18545600 & 1.951667177 & 0.4066124399557611 \\
$\mathrm{Cd}-\mathrm{S}$ & 5.322928773 & 18.66179645 & 3.505926388 & 0.327164494602450 \\
$\mathrm{Cd}-\mathrm{Te}$ & 18.66179645 & 28.76019326 & 1.135231317 & 0.479475162402413 \\
\hline
\end{tabular}

Because that the curve $\varpi(x)$ passes through the origin $(0,0)$, so $\eta_{0} \cong 0$ and $\eta_{1}$ is the tangent slope of linear fitting function $\varpi=\eta_{1} x$ besides $x=0$.

$$
\varpi=\eta_{1} x \Rightarrow \sqrt{\frac{m}{\beta}} \underline{\omega}=\eta_{1} a q,
$$

where $\underline{\omega}$ and $\underline{\beta}$ are calculated by linear fitting process, then

$$
\underline{\omega}=a \eta_{1} \sqrt{\frac{\beta}{m}} q
$$

which we compare with Equation (5), so

$$
\begin{gathered}
\underline{\omega}=a \eta_{1} \sqrt{\frac{\underline{\beta}}{m}} q \cong s q, \text { then } \\
\beta=\frac{m s^{2}}{a^{2} \eta_{1}^{2}} .
\end{gathered}
$$

\subsection{Mathematical Analyzing Proof}

Proceeding from Equation (8), which is

$$
\varpi=\sqrt{\left(1+\frac{1}{p}\right)-\sqrt{1+\frac{1}{p^{2}}+\frac{2}{p} \cos x}} .
$$

A first estimation of the force constant $\beta$ may be derived from simple consideration. Indeed, as $x$ approaches zero, $\cos \theta \cong 1-\frac{x^{2}}{2}$, so, Equation (8) can be rewritten under the following form

$$
\begin{gathered}
\varpi^{2}=\left(1+\frac{1}{p}\right)-\sqrt{1+\frac{1}{p^{2}}+\frac{2}{p}\left(1-\frac{x^{2}}{2}\right)}, \text { around } x=0 \\
\text { or } \varpi^{2}=\left(1+\frac{1}{p}\right)\left[1-\sqrt{1-\frac{x^{2}}{p(1+1 / p)^{2}}}\right],
\end{gathered}
$$

for values of $x$ around zero, we have $\frac{x^{2}}{p(1+1 / p)^{2}} \ll 1$, and, consequently 
(as $(1+y)^{1 / 2} \cong 1+\frac{1}{2} y$ as $\left.y \cong 0\right)$, Equation (14) reduces to the simple form

$$
\varpi^{2}=\frac{x^{2}}{2(p+1)}
$$

as $x=a q, \quad p=\frac{M}{m}(M \geq m$, then $p \geq 1)$ and $\varpi=\sqrt{\frac{m}{\beta}} \omega$, then from Equation 15 , one can obtain the following expression of $\omega$.

$$
\omega=\left(\sqrt{\frac{\beta}{2(M+m)}} a\right) q
$$

According to Equation (5), we get

$$
\begin{aligned}
& s=\left(\sqrt{\frac{\beta}{2(M+m)}} a\right), \\
& \text { then } \beta=\frac{4 \bar{m} s^{2}}{a^{2}},
\end{aligned}
$$

where $\bar{m}=\frac{m+M}{2}$.

\section{Comparison and Discussion}

Comparing the estimated force constant and the analytically extracted one, we get the results shown in Table 2 in which the last column shows the calculated values of $\beta$ according to Equation 18 and we can observe a good concordance between these values and those given by the fitting process according to Equation 12 (in the adjacent column) and, so, as Figure 4 shows.

We can now introduce the interatomic force constant, $\beta$, via the following relation

$$
\beta=\frac{4 \bar{m} s^{2}}{a^{2}}
$$

Table 2. Comparison between the estimated force constant (Equation (12)) and the analytically extracted one (Equation (18)).

\begin{tabular}{cccccc}
\hline Crystal & $\begin{array}{c}a[5] \\
\left(\times 10^{-10} \mathrm{~m}\right)\end{array}$ & $\begin{array}{c}B[11] \\
\left(\times 10^{11}\right)\end{array}$ & $S(\mathrm{~m} / \mathrm{s})$ & $\begin{array}{c}\beta=\frac{m s^{2}}{a^{2} \eta_{1}} \\
\text { Estimated }\end{array}$ & $\begin{array}{c}\beta=\frac{4 \bar{m} s^{2}}{a^{2}} \\
\text { Extracted }\end{array}$ \\
\hline C-C & 3.56 & 4.4000 & 11155.092 & 79.7487862 & 78.31346728 \\
$\mathrm{Si}-\mathrm{Si}$ & 5.43 & 0.9908 & 6520.984 & 27.39779461 & 26.90453009 \\
$\mathrm{Ge}-\mathrm{Ge}$ & 5.66 & 0.8501 & 3998.367 & 24.49891544 & 24.05782553 \\
$\mathrm{Si}-\mathrm{C}$ & 4.35 & 2.1142 & 8084.071 & 47.27084074 & 45.98786306 \\
$\mathrm{Zn}-\mathrm{S}$ & 5.41 & 0.5032 & 3508.744 & 13.95506009 & 13.61016036 \\
$\mathrm{Zn}-\mathrm{Te}$ & 6.10 & 0.4046 & 2673.068 & 12.60025426 & 12.30523188 \\
$\mathrm{Cd}-\mathrm{S}$ & 5.82 & 0.4783 & 2942.99 & 13.84632183 & 13.34362773 \\
$\mathrm{Cd}-\mathrm{Te}$ & 6.48 & 0.3068 & 2288.505 & 10.12465468 & 9.939901797 \\
\hline
\end{tabular}




$$
\begin{aligned}
& \qquad s=\sqrt{\frac{B}{\rho}}=\sqrt{\frac{B v_{c}}{m+M}}, \rho \text { is the density [12], } \\
& v_{c} \text { is the primitive cell volume, such as } v_{c}=\frac{a^{3}}{\underline{n}} \text { [12], }
\end{aligned}
$$

where $\underline{n}$ is the number of atoms in a primary cell; Figure 5 shows the difference between primary cell and primitive cell in B. C. C. structure as an example.

Substituting from Equation (20) in Equation (19) then in Equation (18), we get

$$
\beta=\frac{2}{n} B a,
$$

which is the relation we are looking for.

As an example, for F. C. C. Structure, as $\underline{n}=4, \quad \beta=\frac{1}{2} B a$.

It is worth noting that a similar relation has been shown to be valid for solids with a variety of crystal structures [4].

Comparing Equation (12) and Equation (18), we find

$$
\frac{m s^{2}}{a^{2} \eta_{1}^{2}}=\frac{4 \bar{m} s^{2}}{a^{2}},
$$

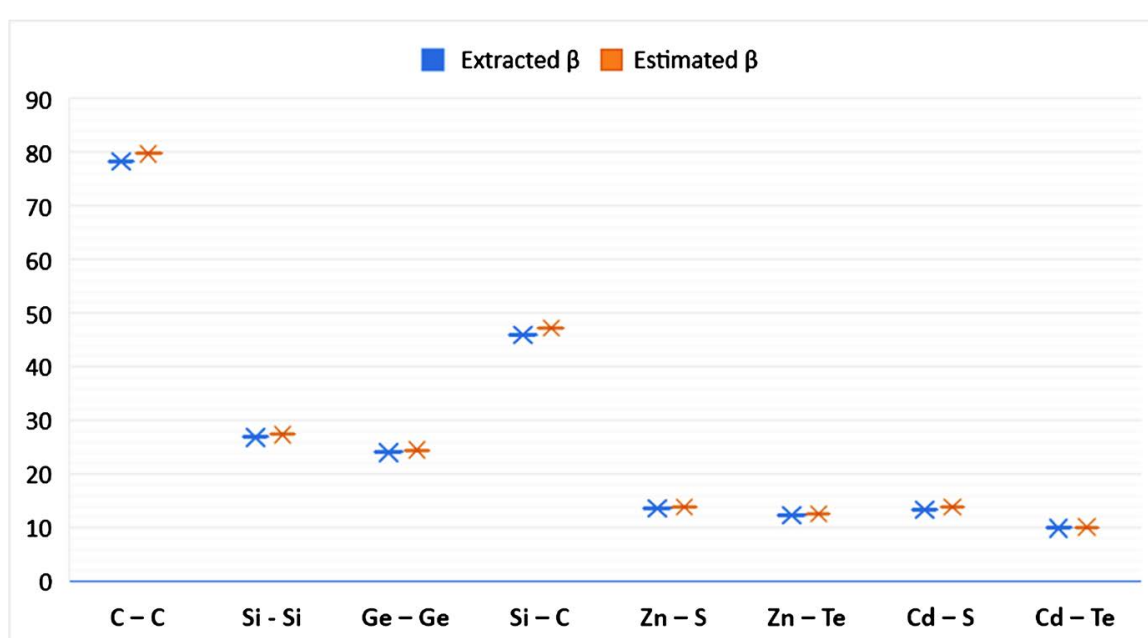

Figure 4. Comparison between the estimated $\beta$ and the analytically extracted $\beta$.

$$
\begin{aligned}
& \underline{n}=1 \text { for S.C. } \\
& \underline{n}=2 \text { for B.C.C. } \\
& n=4 \text { for F.C.C. }
\end{aligned}
$$

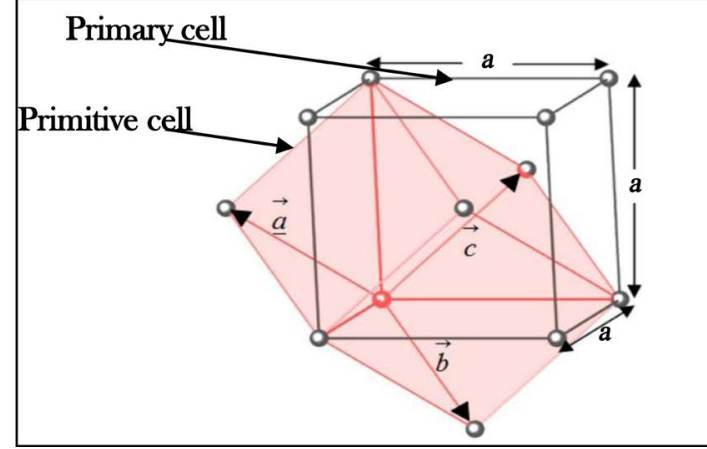

Figure 5. Primary cell and primitive cell in B. C. C. structure [13]. 


$$
\text { then } \frac{m s^{2}}{a^{2} \eta_{1}^{2}}=\frac{4 \bar{m} s^{2}}{a^{2}}
$$

which gives us the exact values of the slope $\eta_{1}$ for the matters used in this work as shown in Table 3, and can be compared to their estimated values in Table 1 which is shown in Figure 6.

As direct results from the obtained force constant relation, we can calculate the maximum acoustic angular frequency $\left(\omega_{a c}\right)_{\max }$, the minimum optical angular frequency $\left(\omega_{o p}\right)_{\min }$ and the maximum optical angular frequency $\left(\omega_{o p}\right)_{\max }$, which can easily be measured experimentally.

From Figure 2, we can see that

$$
\beta=\frac{1}{2} M\left(\omega_{a c}\right)_{\max }^{2}=\frac{1}{2} m\left(\omega_{o p}\right)_{\min }^{2}=\frac{1}{2} \mu\left(\omega_{o p}\right)_{\max }^{2},
$$

where $\mu$ is the reduced mass $\left(\frac{1}{\mu}=\frac{1}{M}+\frac{1}{m}\right)$.

By substituting with $\beta=\frac{2(M+m) s^{2}}{a^{2}}$, we can deduce that

Table 3. Comparison between the extracted $\eta_{1}$ and the estimated $\eta_{1}$ for the used matters.

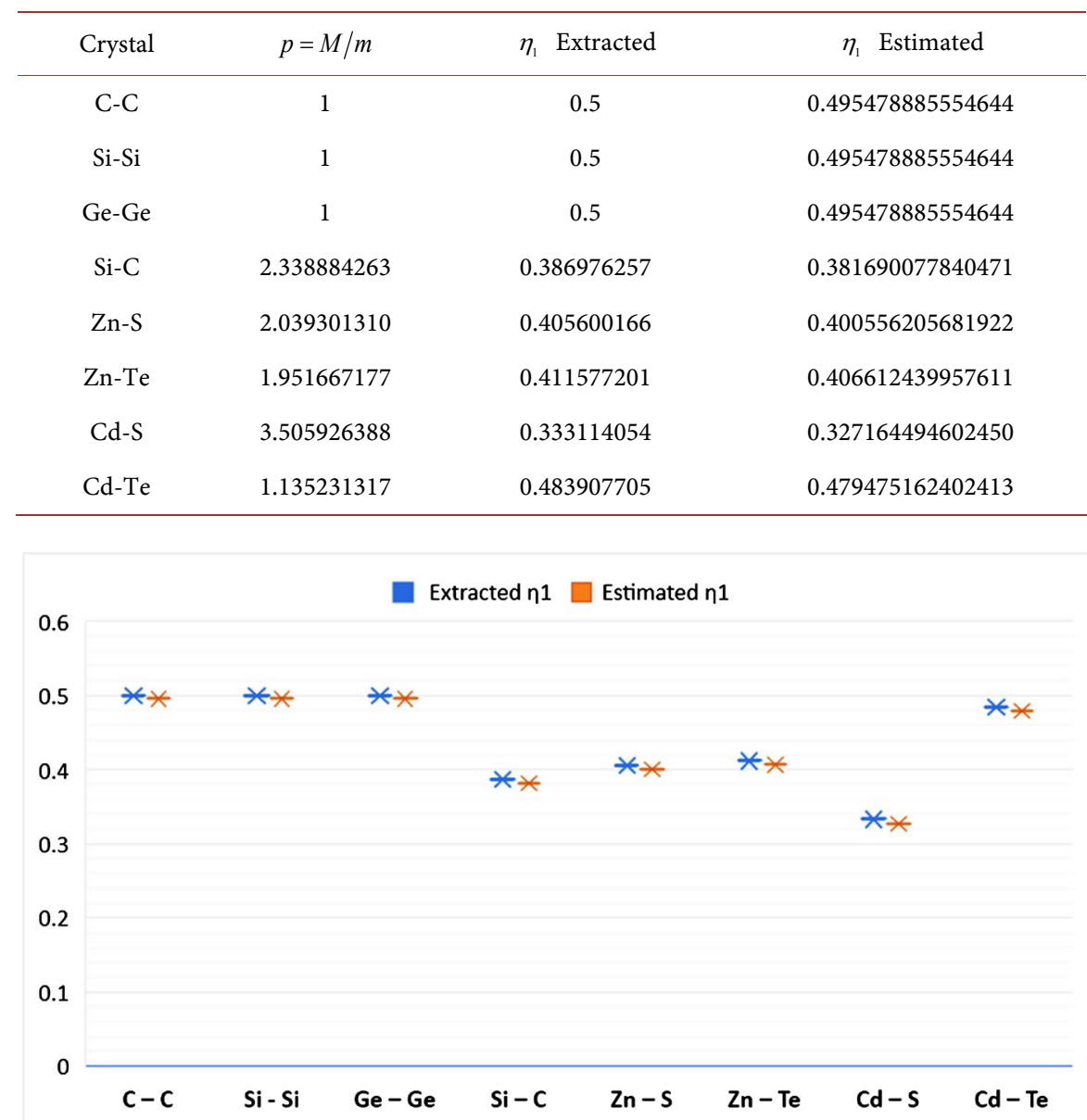

Figure 6. Comparison between the estimated $\eta_{1}$ and the analytically extracted $\eta_{1}$. 
Table 4. Values of $\left(\omega_{a c}\right)_{\max },\left(\omega_{o p}\right)_{\min }$ and $\left(\omega_{o p}\right)_{\max }$ according to the resulted relation of $\beta,\left(\beta=\frac{4 \bar{m} s^{2}}{a^{2}}\right.$, where $\left.\bar{m}=\frac{M+m}{2}\right)$.

\begin{tabular}{ccccccc}
\hline Crystal & $a\left(\times 10^{-10}\right)[5]$ & $p=\frac{M}{m}$ & $S(\mathrm{~m} / \mathrm{s})$ & $\begin{array}{c}\left(\omega_{a c}\right)_{\max } \\
\left(\times 10^{13}\right)\end{array}$ & $\begin{array}{c}\left(\omega_{o p}\right)_{\min } \\
\left(\times 10^{13}\right)\end{array}$ & $\begin{array}{c}\left(\omega_{o p}\right)_{\max } \\
\left(\times 10^{13}\right)\end{array}$ \\
\hline C-C & 3.56 & 1 & 11155.092 & 8.863 & 8.863 & 12.534 \\
$\mathrm{Si}-\mathrm{Si}$ & 5.43 & 1 & 6520.984 & 3.397 & 3.397 & 4.804 \\
$\mathrm{Ge}-\mathrm{Ge}$ & 5.66 & 1 & 3998.367 & 1.998 & 1.998 & 2.826 \\
$\mathrm{Si}-\mathrm{C}$ & 4.35 & 2.338884263 & 8084.071 & 4.441 & 6.792 & 8.115 \\
$\mathrm{Zn}-\mathrm{S}$ & 5.41 & 2.039301310 & 3508.744 & 1.584 & 2.262 & 2.761 \\
$\mathrm{Zn}-\mathrm{Te}$ & 6.10 & 1.951667177 & 2673.068 & 1.078 & 1.506 & 1.852 \\
$\mathrm{Cd}-\mathrm{S}$ & 5.82 & 3.505926388 & 2942.99 & 1.147 & 2.147 & 2.435 \\
$\mathrm{Cd}-\mathrm{Te}$ & 6.48 & 1.135231317 & 2288.505 & 0.831 & 0.885 & 1.214 \\
\hline
\end{tabular}

$$
\begin{aligned}
& \left(\omega_{a c}\right)_{\max }=2 \sqrt{\left(1+\frac{1}{p}\right)} \cdot \frac{s}{a}, \\
& \left(\omega_{o p}\right)_{\min }=2 \sqrt{(1+p)} \cdot \frac{s}{a}, \\
& \text { and }\left(\omega_{o p}\right)_{\max }=\frac{2(1+p)}{\sqrt{p}} \cdot \frac{s}{a},
\end{aligned}
$$

which Table 4 shows their values for the substances of this work.

\section{Conclusions}

The simple approach, detailed in this work, based on linear fitting by computer programming and mathematical analysis, permitted computation of the constant force (CF) as a linear function of the bulk modulus and interatomic distance parameters, under the assumption that only interactions between first nearest neighbors are considered. The two derived relations from the two above-mentioned methods have led to similar results for some mono- and diatomic molecules of the semiconductor type. Furthermore, these two relations enabled us to express the interatomic force constant as proportional to the bulk modulus and the interatomic distance.

The importance of deriving this relation is that many physical quantities are linked to the force constant, the bulk modulus and the interatomic distance and so, it could be a contribution to increase crystal structure understanding and may be used in many other theoretical works.

Moreover, this resulted mathematical relation makes it possible to calculate interatomic Hooke's force constant for solid matters in terms of bulk modulus and interatomic distance, and so to calculate bulk modulus in terms of the others, which can help understanding the relationship between sub-fusion and fusion energies with fusion temperature. 
The results of this work will be used in an attempt to extract mathematical relations between correlation energy, sub-fusion energy and fusion energy with temperature, which will clarify many physical issues and provide broader research possibilities.

\section{Conflicts of Interest}

The authors declare no conflicts of interest regarding the publication of this paper.

\section{References}

[1] Tapia Goazalez, J., Villanueva Lopez, C., Peon-Escalante, R., Quintal, R., Medina, J., Penunuri, F. and Avilves, F. (2015) The Bond Force Constant and Bulk Modulus of Small Fullerenes Using Density Functional Theory and Finite Element Analysis. Journal of Molecular Modeling, 21. https://doi.org/10.1007/s00894-015-2649-6

[2] Santiago-Perez, D.G., de Leon-Perez, F., Mora-Ramos, M.E. and Perez-Alvarez (2008) Force Constants and Dispersion Relations in GaN. PIERS Online, 4, 187-190. https://doi.org/10.2529/PIERS071005121557

[3] Kot, M., Nagahashi, H. and Szymczak, P. (2014) Elastic Moduli of Simple Mass Spring Models. Springer-Verlag, Berlin, Heidelberg. https://doi.org/10.1007/s00371-014-1015-5

[4] Dolocan, V., Dolocan, A. and Dolocan, V.O. (2008) Relation of Interatomic Forces in Solids to Bulk Modulus, Cohesive Energy and Thermal Expansion. Modern Physics Letters B, 22, 2481-2492. https://doi.org/10.1142/S0217984908017096

[5] Kittel, C. (1996) Introduction to Solid State Physics. 7th Edition, John Wiley \& sons, Inc., New York.

[6] Ashcroft, N.W. and Mermin, N.D. (1976) Solid State Physics. Holt Rin, and Win., New York.

[7] Brillouin, L. (1946) Wave Propagation in Periodic Structures: Electric Filters and Crystal Lattices. Originally Published by the McGraw-Hill Book Company, Inc. (Dover Phoenix Editions, Inc., 2003)

[8] Rössler, U. (2009) Solid State Theory: An Introduction. 2nd Revised and Extended Edition, Springer, Dordrecht, Heidelberg, London, New York, Springer-Verlag, Berlin, Heidelberg.

[9] Quinn, J.J. and Yi, K.-S. (2009) Solid State Physics: Principles and Modern Applications. Springer-Verlag, Berlin, Heidelberg.

[10] Semat, H. and Albright, J. (1972) Introduction to Atomic and Nuclear Physics. Holt, Rin. and Win. C., USA.

[11] Adachi, S. (2004) Handbook on Physical Properties of Semiconductors. Vol. 3, II-VI Compound Semiconductors, Kluwer Academic Publishers group, Dordrecht.

[12] Razeghi, M. (2006) Fundamentals of Solid State Engineering. 2nd Edition, Springer Science + Business Media, Evanston.

[13] Elliott, S.R. (1998) The Physics and Chemistry of Solids. John Wiley \& Sons, Ltd., England. 